\title{
Flavor preferences conditioned with starch in rats
}

\author{
ISRAEL RAMIREZ \\ Monell Chemical Senses Center, Philadelphia, Pennsylvania
}

\begin{abstract}
Rats were trained to associate artificial cherry or grape flavors with $1 \%$ starch suspensions. Conditioning was assessed by offering the rats a choice of the cherry versus grape flavors without starch. Conditioned preferences were moderately strong and persistent; 3 days of conditioning produced a preference that did not fully extinguish within 18 days. Food deprivation substantially increased intake of $1 \%$ starch suspension. However, the degree of preference conditioned was not influenced by the availability of food during the conditioning period; the rats that had been food deprived during training acquired as strong a preference as did those fed freely during training. The degree of preference obtained was similar in the rats given reinforcing and nonreinforcing fluids simultaneously or sequentially. Starch conditioned a slightly stronger preference than did the same concentration of glucose, even though these substances contain the same amount of calories. A statistically significant, but weak, preference was conditioned by $0.5 \%$, but not by $0.25 \%$, starch. It is proposed that the flavor of starch, independent of its calories, is reinforcing to rats.
\end{abstract}

Pairing a flavor with a nutrient increases subsequent preference for the flavor (Bolles, 1983; Booth, 1990; Mehiel, 1991; Sclafani, 1990, 1991; Tordoff, 1991; Weingarten, 1990). This form of conditioning is reinforced partly by the hedonic effects of the nutrient's oral-sensory properties (flavor) and partly by the nutrient's postingestive effects (calories). The relative importance of oralsensory and postingestive factors in flavor preference conditioning is unknown. Several studies have shown that nutrients can reinforce flavor preferences through their postingestive effects (Sclafani, 1990, 1991; Tordoff, 1991), but evidence supporting flavor-reinforced conditioning is limited to sweet (Fanselow \& Birk, 1982; Messier \& White, 1984) and greasy (Elizalde \& Sclafani, 1990a) substances.

The world's most abundant nutrient, starch, is neither sweet nor greasy. Little is known about the reinforcing properties of starch because investigators have used maltodextrins as a convenient substitute (Booth, Mather, \& Fuller, 1982; Elizalde \& Sclafani, 1988; Mehiel, 1991; Sclafani \& Nissenbaum, 1988). This substitution is not appropriate because the two substances have very different sensory properties in conditioned-taste-aversion studies (Ramirez, 1991b). Indeed, these substances probably are sensed via entirely different sensory systems, gustation for maltodextrins and touch and olfaction for starch (Ramirez, in press).

In the experiments described here, flavor preferences were conditioned in rats by offering them a choice of two artificially flavored fluids, one of which contained starch.

This work was supported by National Science Foundation Grant BNS 87-19309, NIH Grant 1 R01 DC01107, and the Monell Chemical Senses Center. Highly skilled technical assistance was provided by Mara Ketchum. Address correspondence to I. Ramirez, Monell Chemical Senses Center, Philadelphia, PA 19104-3308.
The degree of preference conditioning was subsequently assessed by offering the rats a choice of the two training flavors without starch. Because there is no noncaloric starch flavor, analogous to saccharin, a variety of indirect methods were used to identify the relative contributions of oral-sensory and postingestive factors. The concentration of starch used was just above the preference threshold (Ramirez, 1991a) in order to minimize postingestive effects.

\section{EXPERIMENT 1}

The caloric effects of dilute nutrients should be more easily perceived and have a greater impact in fooddeprived animals. Because rats with free access to food eat frequently and tend to drink at mealtimes (Kissileff, 1969), any nutrients in their drinking water will become mixed with, and diluted by, substantial amounts of highstarch food in their stomachs. Such effects should not occur in food-deprived rats because their stomachs should be empty when they ingest training fluids. Indeed, food deprivation should increase the drive to ingest caloric substances.

One factor that could complicate this experiment is the observation that pairing flavors with a deprivation state can influence subsequent preference (Capaldi \& Myers, 1982; Capaldi, Sheffer, \& Owens, 1991). In order to minimize such effects, both reinforced and nonreinforced flavors were available simultaneously during each training trial.

\section{Method}

Subjects. Twenty-eight female Fischer F-344 rats (Harlan Sprague-Dawley, Indianapolis, $\mathrm{N})$, weighing $133 \pm 2 \mathrm{~g}(M \pm S E M)$, were housed individually in hanging stainless steel cages and were maintained on a $12: 12-\mathrm{h}$ light:dark cycle at $21^{\circ}-24^{\circ} \mathrm{C}$. The rats were freely fed Purina Laboratory Chow ( $\$ 5001)$ in steel hoppers 
in the rear of the cages, except as indicated below. Purina Laboratory Chow contains about $49 \%$ carbohydrate, most of which is starch. All the animals were maintained in the laboratory for at least 1 week after arrival to permit them to acclimate to their surroundings before testing began.

Starch. Regular (dent) cornstarch was purchased locally (Argo, CPC International, Englewood Cliffs, NJ). Cornstarch is a fairly typical starch with regard to its physical and chemical properties, and with regard to the preference values obtained (Ramirez, 1991a 1993b). Conditioned-aversion experiments indicate that cornstarch has a flavor like that of other starches (Ramirez, 1991b, 1992, 1993a).

Starch suspensions are unstable in plain water. As in previous studies, xanthan gum $(0.3 \%$, Sigma Chemical Co., St. Louis, MO) was added to all training fluids to prevent precipitation of starch (Ramirez, 1991a). The control fluid, which did not contain starch, contained the same concentration of xanthan gum as the fluid containing starch.

Procedure. The procedure was similar to that used in previous experiments (Ramirez, 1991a, 1991d). In the present experiment, the rats were randomly assigned to one of two groups of 14 rats each. Both groups were offered a choice of $1 \%$ cornstarch solution versus vehicle on Days 1,3 , and 6 . Group 1 was food deprived on the days that the training fluids were offered. Group 2 was food deprived for 1 day immediately after each day that the fluids were offered. Although both groups were food deprived for exactly the same amount of time, Group 1 was deprived during training, whereas Group 2 was freely fed during training. For half of the rats, the starch contained $0.05 \%$ Kool-Aid cherry flavor, and the vehicle contained $0.05 \%$ Kool-Aid grape flavor (Sclafani \& Nissenbaum, 1988); the other half of the rats received the reverse combination. The rats received a choice between cherry- and grapeflavored water, beginning on Day 9 of the experiment and continuing for the next 18 days. The rats were given tap water in sipper bot- tles to drink freely when they were not given test fluids. The animals always had some fluid to drink, but they never had both tap water and test fluids at the same time.

In all of the tests, intake was measured in the light part of the light:dark cycle, 3 to $4 \mathrm{~h}$ before the lights went off. Test fluids were available for $24 \mathrm{~h}$ at a time. The left-right position of the starch mixture was reversed in successive tests. Fluids were offered in $120-\mathrm{ml}$ glass jars placed inside the front of the cages. All mixtures were formulated on a weight basis because intake was measured by weighing. Suspensions were made fresh daily with deionized water.

Statistics. Preference was computed as intake of the carbohydrate suspension divided by total fluid intake. A value of $50 \%$ indicates no preference, and $100 \%$ indicates complete preference for carbohydrate suspension. Preference scores for grape and cherry flavors were converted to preference for CS+, that is, the flavor that had been paired with starch (Elizalde \& Sclafani, 1988; Sclafani \& Nissenbaum, 1988). Probability values were computed with $t$ tests.

\section{Results and Discussion}

Food deprivation did not enhance the development of a conditioned flavor preference, although it enhanced consumption of starch during training. The rats that were food deprived while starch was available (Group 1) had a greater preference for starch suspension than did those in Group 2, which had both food and the starch suspension available at the same time $[88 \% \pm 1 \%$ for Group 1 vs. $83 \% \pm 2 \%$ for Group $2 ; t(26)=2.1, p<.05]$. The food-deprived rats drank significantly more fluid than did the fed rats $[37 \pm 3$ for Group 1 vs. $28 \pm 1$ g/day for Group $2 ; t(26)=3.2, p<.005]$. These findings are con-

\section{Condition During Training}
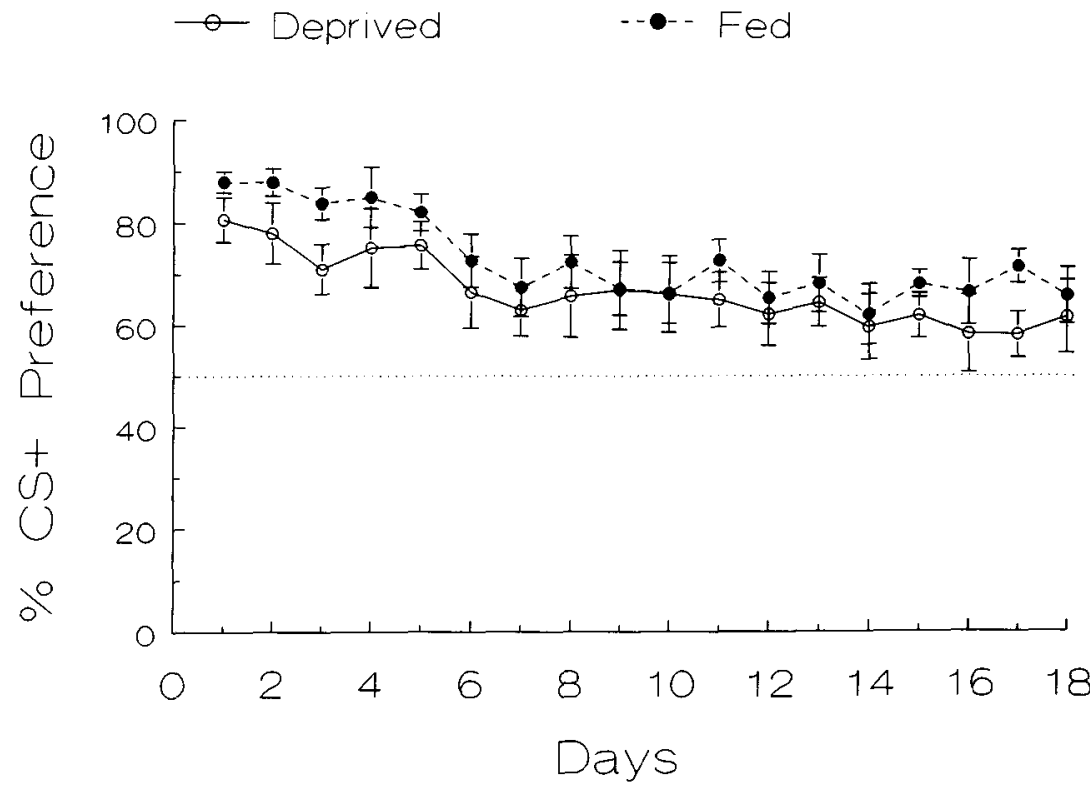

Figure 1. Mean preference for $\mathrm{CS}+$ solutions during extinction by rats trained when food was freely available or when food was withheld in Experiment 1 . All the rats were freely fed during the period depicted in the figure. Each point represents the mean $( \pm S E M)$. The dotted line represents $50 \%$ preference (i.e., no evidence for discrimination). 
sistent with previous studies involving sugars and saccharin (Gilbert \& Sherman, 1970; Smith \& Duffy, 1957).

Figure 1 shows that both groups had strong and persistent preferences for the flavor paired with starch after training. Preference scores for the rats that were trained with both starch and food available at the same time were significantly greater than $50 \%$ (all $t$ tests, $p<.05$ ) on every day except Day 14 of extinction. Preference scores for $\mathrm{CS}+$ for the food-deprived rats in Group 1 were statistically different from $50 \%$ except on Days 10, 12, 14, 16,17 , and 18 . Despite the apparently greater preference for $\mathrm{CS}+$ in Group 2, a mixed-design analysis of variance (group $X$ day) revealed no differences between the two groups $(p s>.20)$.

The observation that food deprivation did not enhance the development of a conditioned flavor preference is consistent with the hypothesis that acquired flavor preference is based on flavor rather than postingestive effects. Furthermore, the degree of preference conditioned and its resistance to extinction were larger than one might expect for a reinforcer containing so few calories. This result is even more surprising when one notes that the animals were tested with a different fluid (plain water) than the one they were trained with (xanthan gum solution); this factor should have facilitated extinction (Bonardi, Honey, \& Hall, 1990). Another group of investigators has reported strong resistance to extinction following conditioning with intragastric infusions (Elizalde \& Sclafani, 1990b), but they used a much longer, more complex training protocol and a more concentrated reinforcer.

\section{EXPERIMENT 2}

If flavor preferences are reinforced by the postingestive effects of starch, offering only one training solution at a time should produce more effective conditioning than offering both rewarded and unrewarded fluids simultaneously (Bolles, Hayward, \& Crandall, 1981; Galef, 1991). Although flavor preferences can be conditioned by postingestive events when rats are offered reinforced and nonreinforced solutions simultaneously (Baker \& Booth, 1989; Drucker, Ackroff, \& Sclafani, 1993), such a procedure should not produce optimal conditioning. The reason for this is that carbohydrate digestion can take many minutes, or even hours (Dreher, Dreher, \& Berry, 1984); a rat that ingests a fluid, and then ingests another before the first is fully digested, should find it difficult to learn which fluid produced the delayed postingestive effect. The influence of this factor should be particularly severe with starch because starch is digested more slowly than are sugars (Malik, Trout, Emamali, \& Brendza, 1982). Experiment 2 was an examination of whether simultaneous presentation of rewarded and unrewarded fluids hinders conditioning.

\section{Method}

Subjects and Apparatus. Thirty-two female F-344 rats, weighing $119 \pm 1 \mathrm{~g}$, were obtained from Harlan Sprague-Dawley and housed as in Experiment 1. Conditioning and testing occurred in each rat's home cage.

Procedure. The rats were assigned randomly to either a sequential $(n=16)$ or a simultaneous group $(n=16)$. The protocol was somewhat complicated because an attempt was made to ensure that the number of training days was the same for both groups. On Days 1,3 , and 5 of training, the sequential group was given a $1 \%$ starch suspension mixed with the appropriate flavor (half of the rats received cherry plus starch; the other half received grape plus starch). The alternate flavor in the absence of starch served as CS- . The simultaneous group was given both the flavored starch suspension and flavored vehicle on Days 1, 3, and 5 of training. On Days 2, 4, and 6, both groups were given the vehicle with the other flavor. All the rats in both groups were given plain water for 3 days after the last conditioning session and then were tested for their preference for CS+ versus CS- in plain water for 2 days. All other aspects of the procedure were the same as in Experiment 1.

\section{Results and Discussion}

The sequential protocol did not enhance conditioning. As shown in Table 1, the sequential group ingested somewhat more starch suspension than vehicle during training, whereas the reverse was true for the simultaneous group. Mean CS+ preference scores during the final test were significantly greater than $50 \%(68 \% \pm 3 \%$ and $65 \% \pm 2 \%$, respectively, for the simultaneous and sequential groups, $p<.001$ ). The groups did not differ from each other with regard to preference or intake on any day or combination of days (all $p \mathrm{~s}>.2$, including the group $x$ extinction day interaction). Preference scores for Day 1

Table 1

Amount of Fluid Ingested During Training in Experiments 2-5

\begin{tabular}{|c|c|c|c|c|}
\hline Group & $\begin{array}{l}\text { Type of } \\
\text { Fluid }\end{array}$ & $\begin{array}{l}\text { Total Fluid } \\
\text { Ingested* }\end{array}$ & $\begin{array}{c}\text { Fluid Ingested/ } \\
\text { Day }\end{array}$ & $\begin{array}{l}\text { Tota } \\
\text { Days }\end{array}$ \\
\hline \multicolumn{5}{|c|}{ Experiment 2} \\
\hline \multirow[t]{2}{*}{ Simultaneous } & $\begin{array}{l}\text { Vehicle } \\
\text { Starch }\end{array}$ & $69 \pm 2$ & $11.4 \pm 0.3$ & 6 \\
\hline & suspension & $60 \pm 2$ & $20.2 \pm 0.6$ & 3 \\
\hline \multirow[t]{2}{*}{ Sequential } & $\begin{array}{l}\text { Vehicle } \\
\text { Starch }\end{array}$ & $58 \pm 2$ & $19.4 \pm 0.5$ & 3 \\
\hline & suspension & $68 \pm 2$ & $22.5 \pm 0.7$ & 3 \\
\hline \multicolumn{5}{|c|}{ Experiment 3} \\
\hline \multirow[t]{2}{*}{ All } & $\begin{array}{l}\text { Vehicle } \\
\text { Starch }\end{array}$ & $15 \pm 2$ & $5.1 \pm 0.6$ & 3 \\
\hline & suspension & $60 \pm 2$ & $20.0 \pm 0.5$ & 3 \\
\hline \multicolumn{5}{|c|}{ Experiment 4} \\
\hline \multirow[t]{2}{*}{ Starch } & $\begin{array}{l}\text { Vehicle } \\
\text { Starch }\end{array}$ & $74 \pm 2$ & $12.3 \pm 0.3$ & 6 \\
\hline & suspension & $62 \pm 3$ & $20.7 \pm 0.9$ & 3 \\
\hline \multirow[t]{2}{*}{ Glucose } & $\begin{array}{l}\text { Vehicle } \\
\text { Glucose }\end{array}$ & $60 \pm 2$ & $12.0 \pm 0.3$ & 5 \\
\hline & solution & $52 \pm 2$ & $17.2 \pm 0.6$ & 3 \\
\hline \multicolumn{5}{|c|}{ Experiment 5} \\
\hline \multirow[t]{2}{*}{$0.5 \%$} & $\begin{array}{l}\text { Vehicle } \\
\text { Starch }\end{array}$ & $115 \pm 3$ & $14.3 \pm 0.4$ & 8 \\
\hline & suspension & $59 \pm 2$ & $14.7 \pm 0.6$ & 4 \\
\hline \multirow[t]{2}{*}{$0.25 \%$} & $\begin{array}{l}\text { Vehicle } \\
\text { Starch }\end{array}$ & $120 \pm 4$ & $15.0 \pm 0.5$ & 8 \\
\hline & suspension & $40 \pm 2$ & $10.1 \pm 0.6$ & 4 \\
\hline
\end{tabular}

*Total gram intake during the entire training period $( \pm S E M)$. 
of extinction did not differ from those obtained on Day 2 of extinction $(p>.08)$.

The observation that the sequential protocol did not enhance learning is consistent with the hypothesis that the reinforcer is flavor rather than postingestive effects. It also should be noted that both the simultaneous and sequential groups preferred the CS+ fluid even though the simultaneous group drank more vehicle than starch suspension, whereas the sequential group drank more starch suspension than vehicle. This outcome indicates that preference for CS + cannot be attributed to the rats' having consumed more of this solution than the solution associated with CS-.

\section{EXPERIMENT 3}

It seemed possible that the simultaneous group in Experiment 2 might have learned about the properties of the CS- fluid on the days when it was the only fluid available. Data from Experiment 1 cannot be compared with that from Experiment 2 because the protocols used in the two experiments were very different. In Experiment 3, rats were trained in a fashion similar to that used in Experiment 2, except for the absence of days in which the nonreinforced fluid was the only one available.

\section{Method}

Subjects and Apparatus. Eighteen female Fischer (F-344) rats, weighing $131 \pm 3 \mathrm{~g}$, were obtained from Harlan Sprague-Dawley and housed as in Experiment 1. Conditioning and testing occurred in each rat's home cage.
Procedure. All the rats received simultaneous access to both flavored starch suspension and flavored vehicle for 3 consecutive days. Then, the subjects were given plain water for 5 days, followed by a preference test for CS+ versus CS- flavors in plain water for 2 days. All other aspects of the procedure were as in Experiment 1.

\section{Results and Discussion}

Simultaneous presentation of rewarded and nonrewarded fluids did not hinder learning. Table 1 shows that the rats ingested considerably more starch suspension than vehicle. Mean CS+ preference during the final test was significantly greater than $50 \%[86 \% \pm 3 \%$, $t(15)=14, p<.001]$. This level of preference is substantially higher than that seen in Experiment 2.

\section{EXPERIMENT 4}

Sugars owe their reinforcing effects to both their flavor and their postingestive effects (Ackroff \& Sclafani, 1991; Fanselow \& Birk, 1982; Messier \& White, 1984). If the reinforcing effects of starch are solely due to its postingestive effects, then starch should be a less effective reinforcer than glucose. Indeed, because uncooked starch is digested more slowly than glucose (Malik et al., 1982), the postingestive effects of starch should be attenuated on account of delayed reinforcement, compared with those of glucose. In Experiment 4, the reinforcing effects of starch and glucose were compared.

The extinction test offered an opportunity to test postingestive conditioning in another way. It has been proposed that food deprivation enhances preferences conditioned

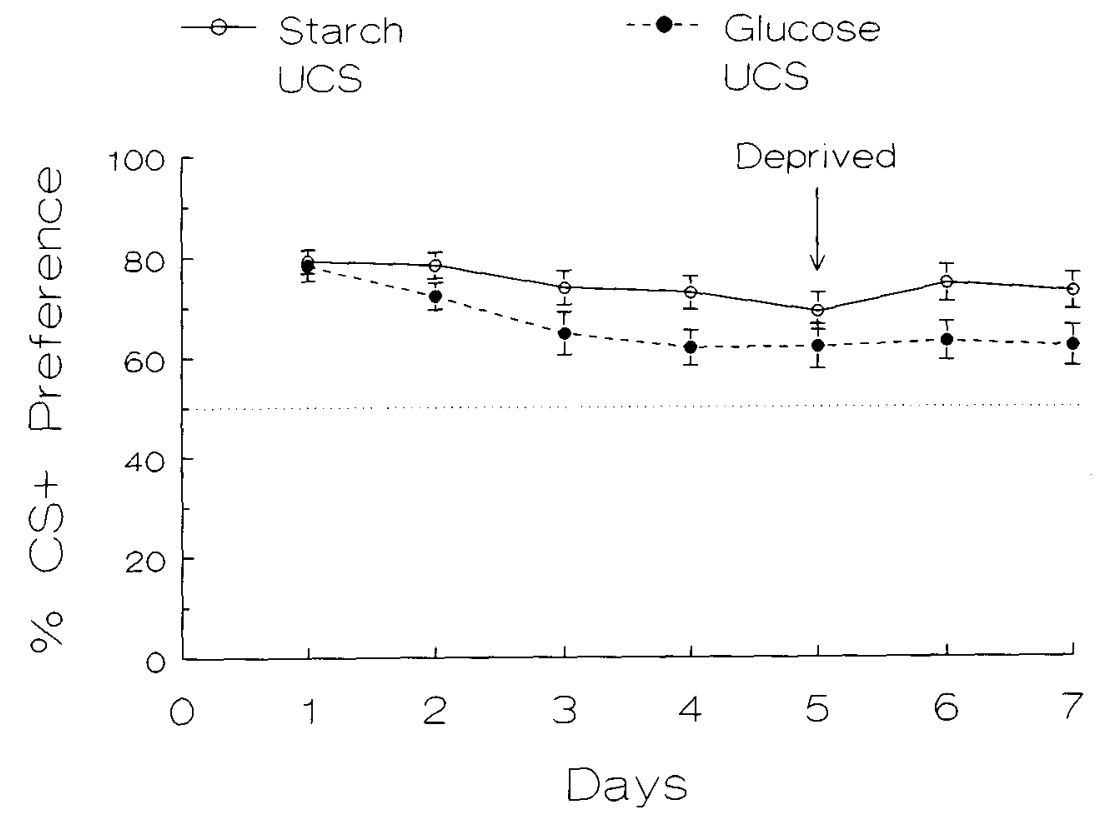

Figure 2. Mean preference for CS+ solutions during extinction by rats trained with $1 \%$ cornstarch or 1\% glucose. Food was always freely available except on Day 5 of extinction, when no food was available. Each point represents the mean $( \pm S E M)$. The dotted line represents $\mathbf{5 0 \%}$ preference (i.e., no evidence for discrimination). 
by calories, but not preferences conditioned by flavor alone (Fedorchak \& Bolles, 1987). Experiment 4 was an investigation of whether withholding food for 1 day during extinction would influence preference.

\section{Method}

Subjects and Apparatus. Thirty-two female F-344 rats, weighing $126 \pm 2 \mathrm{~g}$, were obtained from Harlan Sprague-Dawley and housed as in Experiment 1.

Procedure. The rats were randomly assigned to a starch $(n=16)$ or a glucose $(n=16)$ group. The starch group was trained with $1 \%$ starch, and the glucose group was trained with $1 \%$ glucose (Sigma Chemical Co., St. Louis, MO). The training protocol was the same as that used for the simultaneous group in Experiment 2, except that the glucose group was not offered vehicle on the last day of training; they were given tap water instead. This change was implemented in order to make the ratio of carbohydrate intake to vehicle intake equal in the two groups. If both groups had been offered test fluids for the same number of days, one might be able to argue that the starch group had become more familiar with the $\mathrm{CS}+$ flavor than the CS- flavor.

Extinction tests began 3 days after the last training day for the starch group. The rats were offered grape and cherry flavors in plain water for 7 consecutive days. Food was withheld on Day 5. All other aspects of the procedure were as in Experiment 1.

\section{Results and Discussion}

Starch was a slightly better reinforcer than was glucose. The rats showed a stronger preference for starch $(81 \% \pm 2 \%)$ than for glucose $(73 \% \pm 2 \%)$ during training $[t(30)=3.9, p<.001]$. This result, by itself, suggests that $1 \%$ starch has more potent motivational effects than does equicaloric glucose. The number of training trials was set so that the ratio of CS+ to CS- fluid intake was similar in the two groups $(0.85 \pm 0.04$ and $0.86 \pm 0.03$, respectively, for the starch and glucose groups).

Figure 2 shows that the starch group showed a stronger preference for CS+ than did the glucose group over the entire extinction period $[F(1,30)=5.6, p<.025]$. This result suggests that the reinforcing effects of starch are due to its flavor rather than its calories.

As can be seen in Figure 2, food deprivation had no effect on fluid preference; however, total fluid intake was dramatically reduced. On the day before food deprivation, the starch and glucose groups drank, respectively, $23 \pm 2 \mathrm{~g}$ and $21 \pm 1 \mathrm{~g}$. On the day food was withheld, the starch and glucose groups drank, respectively, only $9 \pm 1 \mathrm{~g}$ and $10 \pm 1 \mathrm{~g}$. This outcome is unlike the pattern seen during training in Experiment 1. These results are consistent with the hypothesis that the reinforcer is flavor rather than postingestive effects.

\section{EXPERIMENT 5}

The final experiment was an attempt to determine whether concentrations lower than $1 \%$ starch can be rewarding. Although there is no starch concentration having zero postingestive effects, explanations based on postingestive effects become less plausible as the concentration is decreased.

\section{Method}

Subjects and Apparatus. Thirty-two female F-344 rats, weighing $104 \pm 1 \mathrm{~g}$, were obtained from Harlan Sprague-Dawley and housed as in Experiment 1.

Procedure. The rats were randomly assigned to one of two groups of 16 rats each. One group was trained with $0.5 \%$ starch and the other group was trained with $0.25 \%$ cornstarch. Both groups were trained by a modification of the procedure that was used for the simultaneous group in Experiment 2. The rats received a choice of flavored starch suspension and vehicle on Days 1, 3, 5, and 7, and flavored vehicle alone on Days 2, 4, 6, and 8. Training was interrupted between Days 4 and 5 by a 3-day rest period (a weekend), during which the rats were given plain water to drink. Beginning 5 days after the final training day, the rats were offered a choice of cherry-versus grape-flavored water for 2 days. All other aspects of the procedure were as in Experiment 1.

\section{Results and Discussion}

Only one of the two starch concentrations elicited a significant preference during training; this concentration also produced significant conditioning. As would be expected, starch preference was lower during training than in the previous experiments (Group 0.5\%, 62\% $\pm 2 \%$; Group $0.25 \%, 53 \% \pm 3 \%$ ). This difference between groups was statistically significant $[t(30)=2.6, p<.02]$. Mean starch preference for Group $0.5 \%$ was significantly greater than $50 \%[t(15)=5.5, p<.001]$. As shown in Table 1, all the rats drank substantially more of the nonreinforced flavor than the starch flavor because preferences were very low.

Mean CS+ preference during the final test in Group $0.5 \%$ was significantly greater than $50 \%[58 \% \pm 3 \%$, $t(15)=2.5, p<.025]$. Mean CS+ preference in Group $0.25 \%$ was not significantly greater than $50 \%$ $(53 \% \pm 4 \%)$. Therefore, the lowest concentration that can be conditioned with this technique was approximately $0.5 \%$. Because rats of this age and strain ingest roughly $30 \mathrm{kcal}$ of food per day (Ramirez, 1991c) and less than $15 \mathrm{ml}$ of $0.5 \%$ starch suspension per day (see Table 1 ), the rats were getting about $1 \%$ of their daily calories from the starch suspension. It is therefore remarkable that conditioning was detectable at all.

\section{GENERAL DISCUSSION}

A $1 \%$ starch suspension can condition a moderately strong and persistent flavor preference; 3 days of training conditioned a preference that had not fully extinguished over 18 days. Although the present studies cannot rule out postingestive reinforcement, the predominant reinforcer at low concentrations seems to be flavor rather than calories. This explanation is consistent with the observation that starch is a more effective reinforcer than glucose (Experiment 4). Because glucose owes its reinforcing properties to a mixture of taste and calories, and because glucose has the same number of calories as starch, the flavor of starch must possess reinforcing properties analogous to those exerted by sweet taste. Supporting evidence comes from the observation that the magnitude of 
the conditioned preference was unaffected by the availability of food during training (Experiment 1) and testing (Experiment 4). Furthermore, it would be difficult to account for the ability of rats to learn preferences with concurrent presentation of rewarding and unrewarding fluids, unless the conditioning was assumed to be reinforced by flavor (Experiments 2 and 3 ).

One technical problem with the present experiments is that untrained rats tend to ingest starch suspensions more avidly than they drink vehicle. There is no simple way to control for this. If one limits the amount of time that each fluid is available, rats will tend to drink more starch suspension than vehicle. If one limits the amount of fluid available, rats will drink the starch suspension first and then ingest the vehicle. The approach taken in the present studies was to vary the availability of the fluids, so that in some experiments the rats drank more starch than vehicle, whereas in other experiments they drank more vehicle than starch, as is shown in Table 1. It seems reasonable to conclude that the differential intake of starch and vehicle cannot account for the consistent preferences for the flavor paired with starch, seen in all the experiments.

In the present experiments, only low concentrations of a single variety of starch were examined. These results probably apply to other varieties and concentrations of starch because rats prefer several different starches to a similar degree (Ramirez, 1991a). Furthermore, conditioned-aversion studies indicate that botanically diverse starches have similar chemosensory properties (Ramirez, 1991b, 1992, 1993a). Available data indicate that when given a choice of two concentrations of starch, rats usually prefer the more concentrated starch suspension (Ramirez, unpublished data). Therefore, if 0.5\%-1\% starch can act as a reinforcer, higher concentrations must also be able to reinforce fluid preferences.

The majority of recent discussions of flavor preference conditioning have emphasized the postingestive effects of foods (Booth, 1990; Mehiel, 1991; Sclafani, 1990, 1991; Tordoff, 1991; Tordoff, Tepper, \& Friedman, 1987; Weingarten, 1990). A few researchers have emphasized social factors (Galef, 1991; Rozin, 1990). The present results suggest that more attention should be given to the rewarding chemosensory effects of nutrients.

\section{REFERENCES}

ACKROFF, K., \& SCLAFANI, A. (1991). Flavor preferences conditioned by sugars: Rats learn to prefer glucose over fructose. Physiology \& Behavior, 50, 815-824.

BAKer, B. J., \& BoOTH, D. A. (1989). Preference conditioning by concurrent diets with delayed proportional reinforcement. Physiology \& Behavior, 46, 585-590.

Bolles, R. C. (1983). A 'mixed' model of taste preference. In R. Mellgren (Ed.), Animal cognition and behavior (pp. 65-82). Amsterdam: North-Holland.

Bolles, R. C., Hayward, L., \& Crandall, C. (1981). Conditioned taste preferences based on caloric density. Journal of Experimental Psychology, 7, 59-70.
Bonardi, C., Honey, R. C., \& Hall, G. (1990). Context specificity of conditioning in flavor-aversion learning: Extinction and blocking tests. Animal Learning \& Behavior, 18, 229-237.

Bоотн, D. A. (1990). Learned role of tastes in eating motivation. In E. D. Capaldi \& T. L. Powley (Eds.), Taste, experience, and feeding (pp. 179-194). Washington: American Psychological Association.

Booth, D. A., Mather, P., \& Fuller, J. (1982). Starch content of ordinary foods associatively conditions human appetite and satiation, indexed by intake and eating pleasantness of starch-paired flavors. Appetite, 3, 163-184.

CAPALDI, E. D., \& MYERS, D. E. (1982). Taste preferences as a function of food deprivation during original taste exposure. Animal Learning \& Behavior, 10, 211-219.

Capaldi, E. D., Sheffer, J., \& OWens, J. (1991). Food deprivation and conditioned flavor preferences based on sweetened and unsweetened foods. Animal Learning \& Behavior, 19, 361-368.

Dreher, M. L., Dreher, C. J., \& Berry, J. W. (1984). Starch digestibility of foods: A nutritional perspective. CRC Critical Reviews in Food Science \& Nutrition, 20, 47-71.

DRUCKER, B. D., ACKRofF, K., \& SClafani, A. (1993). Flavor preference produced by intragastric Polycose infusions in rats using a concurrent conditioning procedure. Physiology \& Behavior, 54, 351-355.

ElizaLDE, G., \& SClaFANI, A. (1988). Starch-based conditioned flavor preference in rats: Influence of taste, calories and CS-US delay. Appetite, 11, 179-200

Elizalde, G., \& Sclafani, A. (1990a). Fat appetite in rats: Flavor preferences conditioned by nutritive and non-nutritive oil emulsions. Appetite, 15, 189-197.

Elizalde, G., \& SClafan, A. (1990b). Flavor preferences conditioned by intragastric polycose infusions: A detailed analysis using an electronic esophagus preparation. Physiology \& Behavior, 47, 63-77.

FANSELOW, M. S., \& BIRK, J. (1982). Flavor-flavor associations induce hedonic shifts in taste preference. Animal Learming \& Behavior, 10, 223-228.

FedorCHAK, P. M., \& Bolles, R. C. (1987). Hunger enhances the expression of calorie- but not taste-mediated conditioned flavor preferences. Journal of Experimental Psychology: Animal Behavior Processes, 13, 73-79.

GALEF, B. G. J. (1991). A contrarian view of the wisdom of the body as it relates to dietary self-selection. Psychological Bulletin, 98, 218-223.

Gilbert, R. M., \& Sherman, I. P. (1970). Palatability-induced polydipsia: Saccharin, sucrose, and water intake in rats, with and without food deprivation. Psychological Reports, 27, 319-325.

KIssiLEFF, H. (1969). Oropharyngeal control of prandial drinking. Journal of Comparative \& Physiological Psychology, 67, 309-319.

Malik, M. A., Trout, D. L., Emamali, B. C., \& Brendza, D. J. (1982). Intraintestinal carbohydrate in rats during the feeding of glucose or of raw or cooked cornstarch. Proceedings of the Society for Experimental Biology \& Medicine, 169, 80-85.

MeHIEL, R. (1991). Hedonic-shift conditioning with calories. In E. D. Capaldi \& T. L. Powley (Eds.), Taste, experience, and feeding (pp. 107-126). Washington: American Psychological Association.

Messier, C., \& WhITE, N. M. (1984). Contingent and non-contingent actions of sucrose and saccharin reinforcers: Effects on taste preference and memory. Physiology \& Behavior, 32, 195-203.

RamiReZ, I. (1991a). Chemoreception for an insoluble nonvolatile substance: Starch taste? American Journal of Physiology, 260, 192-199.

Ramirez, I. (1991b). Does starch taste like Polycose? Physiology \& Behavior, 50, 389-392.

Ramirez, I. (1991c). High-fat diets stimulate transient hyperphagia whereas wet diets stimulate prolonged hyperphagia in Fischer rats. Physiology \& Behavior, 49, 1223-1228.

Ramirez, I. (1991d). Starch flavor: Apparent discrimination between amylopectin and amylose by rats. Physiology \& Behavior, 50, 1181-1186.

RAMIREZ, I. (1992). Is starch flavor unitary? Evidence from studies of cooked starch. Physiology \& Behavior, 52, 535-540.

RAMIREZ, I. (1993a). Rats discriminate between starch and other substances having a similar texture. Physiology \& Behavior, 53, 373-377. 
RAmiREZ, I. (1993b). Relative preference for starch and sugar in rats. Physiology \& Behavior, 54, 1195-1200.

RAmirez, I. (in press). The role of olfaction in starch and oil preference. American Journal of Physiology.

Rozin, P. (1990). Acquisition of stable food preferences. Nutrition Reviews, 48, 106-112.

SCLAFANI, A. (1990). Nutritionally based learned flavor preferences in rats. In E. D. Capaldi \& T. L. Powley (Eds.), Taste, experience, and feeding (pp. 139-156). Washington: American Psychological Association.

SClafani, A. (1991). Conditioned food preferences. Bulletin of the Psychonomic Sociery, 29, 256-260.

SClafani, A., \& NisSenbaum, J. W. (1988). Robust conditioned flavor preference produced by intragastric starch infusions in rats. American Journal of Physiology, 255, 672-675.

Smith, M., \& DUFFY, M. (1957). Consumption of sucrose and saccharin by hungry and satiated rats. Journal of Comparative \& Physiological Psychology, 50, 65-69.
ToRDOFF, M. G. (1991). Metabolic basis of learned food preferences. In M. I. Friedman, M. G. Tordoff, \& M. R. Kare (Eds.), Chemical senses: Vol. 4. Appetite and nutrition (pp. 239-260). New York: Marcel Dekker.

Tordoff, M. G., TePPer, B. J., \& Friedman, M. I. (1987). Food flavor preferences produced by drinking glucose and oil in normal and diabetic rats: Evidence for conditioning based on fuel oxidation. Physiology \& Behavior, 41, 481-487.

WeINGARTEN, H. P. (1990). Learning, homeostasis, and the control of feeding behavior. In E. D. Capaldi \& T. L. Powley (Eds.), Taste, experience, and feeding (pp. 14-27). Washington: American Psychological Association.

(Manuscript received March 24, 1993; revision accepted for publication August 26, 1993.) 